\title{
Amniocentesis and chorionic villus sampling in HIV-infected pregnant women: a multicentre case series
}

\author{
M Floridia, ${ }^{a}$ G Masuelli, ${ }^{b}$ A Meloni, ${ }^{c}$ I Cetin, ${ }^{d}$ E Tamburrini, ${ }^{e}$ AF Cavaliere, ${ }^{f}$ S Dalzero, ${ }^{g}$ \\ M Sansone, ${ }^{\text {h S Alberico, }}$ B Guerra, ${ }^{\mathrm{j}}$ A Spinillo, ${ }^{\mathrm{k}}$ M Chiadò Fiorio Tin, ${ }^{\mathrm{b}}$ M Ravizza, ${ }^{\mathrm{g}}$ on behalf of \\ The Italian Group on Surveillance on Antiretroviral Treatment in Pregnancy* \\ ${ }^{a}$ Department of Therapeutic Research and Medicines Evaluation, Istituto Superiore di Sanità, Rome, Italy ${ }^{b}$ Department of Obstetrics and \\ Neonatology, Città della Salute e della Scienza Hospital, University of Turin, Turin, Italy ${ }^{c}$ Division of Gynaecology and Obstetrics, S. \\ Giovanni di Dio Hospital, University of Cagliari, Cagliari, Italy ${ }^{\mathrm{d}}$ Department of Obstetrics and Gynaecology, Luigi Sacco Hospital, University \\ of Milan, Milan, Italy ${ }^{\mathrm{e}}$ Department of Infectious Diseases, Catholic University, Rome, Italy ${ }^{\mathrm{f}}$ Department of Obstetrics \& Gynaecology, \\ Catholic University, Rome, Italy ${ }^{g}$ Department of Obstetrics and Gynaecology, DMSD San Paolo Hospital Medical School, University of \\ Milan, Milan, Italy ${ }^{\mathrm{h}}$ Department of Neurosciences, Reproductive and Dentistry Science, University Federico II, Naples, Naples, Italy ${ }^{\mathrm{i}}$ Institute \\ for Maternal and Child Health, IRCCS Burlo Garofolo, Trieste, Italy ${ }^{j}$ St. Orsola-Malpighi General Hospital, University of Bologna, Bologna, \\ Italy ${ }^{k}$ Department of Obstetrics and Gynaecology, IRCCS Policlinico San Matteo, University of Pavia, Pavia, Italy \\ Correspondence: M Floridia, Department of Therapeutic Research and Medicines Evaluation, Istituto Superiore di Sanità, Viale Regina Elena \\ 299, Rome 00161, Italy. Email marco.floridia@iss.it
}

Accepted 16 May 2016. Published Online 20 June 2016.

\begin{abstract}
Objectives To assess in pregnant women with HIV the rates of amniocentesis and chorionic villus sampling (CVS), and the outcomes associated with such procedures.
\end{abstract}

Design Observational study. Data from the Italian National Program on Surveillance on Antiretroviral Treatment in Pregnancy were used.

Setting University and hospital clinics.

Population Pregnant women with HIV.

Methods Temporal trends were analysed by analysis of variance and by the Chi-square test for trend. Quantitative variables were compared by Student's $t$-test and categorical data by the Chisquare test, with odds ratios and $95 \%$ confidence intervals calculated.

Main outcome measures Rate of invasive testing, intrauterine death, HIV transmission.

Results Between 2001 and 2015, among 2065 pregnancies in women with HIV, 113 (5.5\%) had invasive tests performed. The procedures were conducted under antiretroviral treatment in 99 cases $(87.6 \%)$, with a significant increase over time in the proportion of tests performed under highly active antiretroviral therapy (HAART) (100\% in 2011-2015). Three intrauterine deaths were observed $(2.6 \%)$, and 14 pregnancies were terminated because of fetal anomalies. Among 96 live newborns, eight had no information available on HIV status. Among the remaining 88 cases with either amniocentesis $(n=75)$, CVS $(n=12)$, or both $(n=1)$, two HIV transmissions occurred (2.3\%). No HIV transmission occurred among the women who were on HAART at the time of invasive testing, and none after 2005.

Conclusions The findings reinforce the assumption that invasive prenatal testing does not increase the risk of HIV vertical transmission among pregnant women under suppressive antiretroviral treatment.

Keywords Amniocentesis, birth defects, chorionic villus sampling, HIV, invasive testing, mother-to child HIV transmission, pregnancy, prenatal diagnosis.

Tweetable abstract No HIV transmission occurred among women who underwent amniocentesis or CVS under effective anti-HIV regimens.

Linked article This article is commented on by C Eppes, p. 1224 in this issue. To view this mini commentary visit http:// dx.doi.org/10.1111/1471-0528.14194.

Please cite this paper as: Floridia M, Masuelli G, Meloni A, Cetin I, Tamburrini E, Cavaliere AF, Dalzero S, Sansone M, Alberico S, Guerra B, Spinillo A, Chiadò Fiorio Tin M, Ravizza M. Amniocentesis and chorionic villus sampling in HIV-infected pregnant women: a multicentre case series. BJOG 2017; 124:1218-1223.

${ }^{\star}$ Members of the "Italian Group on Surveillance on Antiretroviral Treatment in Pregnancy" are available in Appendix 1. 


\section{Introduction}

The widespread application of effective preventive measures that minimise the risk of HIV transmission from HIVinfected mothers to their children, has levelled rates of transmission at $0.5-2.0 \%{ }^{1,2}$ and increased pregnancy rates and family planning among people with HIV. ${ }^{3}$ Within this context, prenatal diagnosis of congenital abnormalities and chromosomal anomalies represents an issue of growing relevance. In the early decades of HIV epidemic, invasive diagnostic procedures that facilitate fetal exposure to maternal blood, such as amniocentesis and chorionic villus sampling, were usually discouraged, assuming an increased risk of vertical transmission associated with such procedures. ${ }^{4,5}$ In more recent studies, conducted against a background of maternal antiretroviral treatment, rates of transmission were lower, and currently available evidence suggests minimal or no additional risk of transmission if the procedure is performed under treatment with effective combination regimens (highly active antiretroviral therapy, HAART). Most of the published studies are, however, based on a limited number of cases, and there is a need to further explore the impact of invasive procedures on both HIV transmission and pregnancy outcomes. ${ }^{6-10}$

To provide further information on this issue, we analysed data from a large national cohort of pregnant women with HIV, examining rates of invasive testing and outcomes associated with the procedures.

\section{Methods}

Data from the National Program on Surveillance on Antiretroviral Treatment in Pregnancy, which represents the main cohort of HIV-infected pregnant women in Italy, were used. ${ }^{11}$ This is a national observational study of pregnant women with HIV established in Italy in 2001 that covers, based on available prevalence data, ${ }^{12}$ roughly $30-40 \%$ of live births from women with HIV in Italy. Only HIV-positive pregnant women are included, and no specific guidance is given in terms of treatment of HIV infection or prophylaxis for mother-to-child transmission, which are decided by the treating physician. Ethics approval was obtained on 28 September 2001 from the Ethics Committee of the I.N.M.I. Lazzaro Spallanzani in Rome (ref. deliberation no. 578). Major birth defects were defined according to the Antiretroviral Pregnancy Registry definition ${ }^{13}$ and grouped as chromosomal anomalies or major structural defects. Antiretroviral treatment was classified, according to the number of drugs in the regimen, as monotherapy, dual therapy or HAART (three or more drugs, excluding lowdose ritonavir).

Invasive antenatal tests were defined as amniocentesis and chorionic villus sampling (CVS); no specific study protocol was issued for these procedures, given the presence of reference national guidelines for the general population $^{14}$ and specific recommendations for women with HIV. ${ }^{15}$ Non-invasive screening tests for chromosomal anomalies were defined by the following: ultrasonographic assessment of nuchal translucency (usually conducted between 10 and 13 completed weeks of pregnancy); first trimester serum levels of pregnancy-associated plasma protein A (PAPP-A) and free $\beta$-hCG (between 10 and 13 weeks); second-trimester serum levels of alpha-fetoprotein (AFP), $\beta$-hCG and unconjugated oestriol (uE3) (between 14 and 20 weeks). The standard risk cut-off defining positivity of non-invasive tests was $1 / 250 .^{14}$

The study collected data since December 2001. For the current analysis, the study period (2001-2015) was divided in three intervals of 5 years each (2001-2005, 2006-2010, 2011-2015). Temporal trends were analysed by one-way analysis of variance and by the Chi-square test for trend. Quantitative variables were compared by Student's $t$-test and categorical data by the Chi-square test, with odds ratios (OR) and 95\% confidence intervals (CI) calculated. $P$-values $<0.05$ were considered significant. All statistical analyses were performed with the SPSS software, version 22 (IBM Corp., Released 2013, Armonk, NY, USA).

\section{Results}

As of 17 April 2015, following exclusion of pregnancies ending in miscarriage or termination in the first 8 weeks of pregnancy, 2065 pregnancies had available information on pregnancy outcome (77 miscarriages before 22 weeks, 91 voluntary pregnancy terminations, 24 stillbirths at or after 22 weeks, and 1873 pregnancies ending in one or more live births).

Among these pregnancies, 113 (5.5\%) had invasive tests (CVS or amniocentesis) performed in 18 different clinical centres. Two women with pregnancy at risk had two sequential invasive tests in the same pregnancy (two amniocenteses in the first case and a CVS followed by amniocentesis in the other), for a total of 115 tests performed (amniocentesis 97, CVS 18).

The mean age of the study population remained substantially unchanged during the entire period of observation; conversely, the rate of non-invasive tests for chromosomal anomalies increased significantly in more recent years $(P<0.001$, Table 1$)$. The rate of invasive testing in pregnancy (overall 5.5\%) remained stable during the study period, but the proportion of women who were on HAART at the time of amniocentesis or CVS increased significantly, reaching $100 \%$ in $2011-2015(P=0.001$, Table 1$)$.

Having a first antenatal visit in the first 18 weeks of pregnancy increased significantly the likelihood of both non-invasive screening tests (OR 4.411, 95\% CI 3.088- 
Floridia et al.

Table 1. Temporal trends in age, testing, treatment status at invasive testing and HIV transmission (2001-2015)

\begin{tabular}{lccccc}
\hline & All (2001-2015) & 2001-2005 & 2006-2010 & 2011-2015 & P-value \\
\hline Age (years, mean) $(n=2052)$ & 32.2 & 31.9 & 32.5 & 32.1 & $0.107^{*}$ \\
Invasive tests in pregnancy, \% $(n=2065)$ & $5.5(113 / 2065)$ & $5.2(51 / 975)$ & $6.1(53 / 872)$ & $4.1(9 / 218)$ & $0.933^{* *}$ \\
Non-invasive tests in pregnancy, \% $(n=2065)$ & $47.9(799 / 1667)$ & $41.2(327 / 794)$ & $54.1(379 / 701)$ & $54.1(93 / 172)$ & $<0.001^{* *}$ \\
Invasive tests on ARV, \% $(n=113)$ & $87.6(99 / 113)$ & $78.4(40 / 51)$ & $94.3(50 / 53)$ & $100.0(9 / 9)$ & $0.009^{* *}$ \\
Invasive tests on HART, \% $(n=112)$ & $76.8(86 / 112)$ & $62.7(32 / 51)$ & $86.5(45 / 52)$ & $100.0(9 / 9)$ & $0.001^{* *}$ \\
HIV transmission, \% $(n=88)$ & $2.3(2 / 88)$ & $5.4(2 / 37)$ & $0(0 / 44)$ & $0(0 / 7)$ & $0.130^{* *}$ \\
\hline *ANOVA. & & & &
\end{tabular}

6.302, $P<0.001$ ), and invasive diagnostic procedures in pregnancy (OR 2.961, 95\% CI 1.363-6.430, $P=0.006$ ). In 25 cases, invasive tests followed a positive non-invasive screening test (biochemical markers 15; nuchal translucency 10). The invasive test confirmed chromosomal anomalies in $2 / 15(13.3 \%)$ of biochemical-positive (PAPP-A and free $\beta$-HCG) cases (both trisomy 21), and in 3/10 (30\%) of nuchal translucency-positive cases (trisomy 21: 2, trisomy 18: 1).

Among 113 pregnancies with invasive testing, three intrauterine deaths were observed $(2.6 \%)$, all in women who had second trimester amniocentesis performed: in two of them, which occurred in the third trimester (week 26 and 36, respectively), maternal or fetal conditions potentially implicated in the death were present (HELLP syndrome, and trisomy 21 associated with structural defects, respectively). In the third case, intrauterine death occurred at week 19 because of severe chorioamnionitis without any maternal disease or fetal defect. The woman had started an HAART-based regimen (zidovudine, lamivudine, lopinavir/ ritonavir) at week 13 .

In 14 pregnancies, invasive testing was followed by elective termination of pregnancy for fetal anomaly (ETOPFA). In 11 of these cases the invasive test had shown chromosomal anomalies (Trisomy 18: 5; Trisomy 21: 4) or homozygotic haemoglobin disorders (sickle-cell disease: 1; thalassaemia: 1). In the other three cases the results of invasive testing for chromosomal anomalies was either negative (2) or unknown (1), but ultrasonography had shown major structural defects (hydrocephalus: 1, cystic hygroma: 1, bilateral arm hypodysplasia: 1).

Ninety-six pregnancies ended with singleton live births: among the infants, seven $(7.3 \%, 95 \%$ CI 2.1-12.5) had major birth defects: one chromosomal anomaly detected antenatally by amniocentesis/karyotype (Trisomy $\mathrm{X}$ ), and six structural defects, four detected antenatally by ultrasonography: bilateral club foot (2), labiopalatoschisis (1), septal cardiac defect (1), and two detected at birth: polydactyly (1) and atrial septal defect with pulmonary hypertension (1). Overall, among 113 cases with any pregnancy outcome, invasive testing allowed 11 chromosomal anomalies to be detected (9.7\%).

Among 95 live births with information on mode of delivery, only one was delivered vaginally (1.1\%). For 25 pregnancies in the cohort, HIV transmission could not be evaluated either because of termination of pregnancy/intrauterine death $(n=17)$ or because of infant loss to follow up/unavailability of virological data $(n=8)$. Among 88 women with invasive test performed and live births with known HIV status, two cases of HIV transmission were observed $(2.3 \%, 95 \%$ CI $0-5.4 \%)$. Neither of these women were on HAART at the time of invasive testing, whereas all the remaining 86 non-transmitting women were (amniocentesis 78, CVS 8). The two cases of transmission occurred in 2003 and 2005, respectively, and no cases were observed after 2005. In the first case, the mother, an heterozygotic carrier of beta-thalassaemia, was HIV-negative at entry in pregnancy, had CVS at first trimester, and seroconverted for HIV during pregnancy, with HIV infection diagnosed at third trimester and antiretroviral treatment (lamivudine plus zidovudine) started at week 34, with no available information on HIV viral load in pregnancy or at delivery. In the second case, the mother was 41 years old at conception, started zidovudine monotherapy at week 14, had amniocentesis at second trimester under antiretroviral treatment, with viral load levels of 2300 and 5300 copies $/ \mathrm{ml}$ at second and third trimester, respectively. Both cases had elective caesarean delivery, intrapartum administration of intravenous zidovudine, and postnatal infant prophylaxis with zidovudine for 6 weeks.

\section{Discussion}

\section{Main findings}

We assessed, in a large national series of pregnant women with HIV, the rate of invasive testing in pregnancy and the outcomes associated with such procedures. Rate of intrauterine death was low and no cases of HIV 
transmission occurred when invasive tests were performed under HAART coverage.

\section{Strengths and limitations}

We were unable to evaluate formally whether amniocentesis or CVS increases the risk of HIV transmission. This limitation originates from sample size issues and minimal event rate in the presence of universal treatment administration in pregnancy. The case series evaluated, despite being one of the largest reported in women with HIV, remains therefore susceptible to type 2 (false negative) error, particularly for CVS, (with only 13 cases evaluated). The absence of transmission among women on HAART is nonetheless reassuring. The strengths of the present study include the relatively large numbers, the multicentre observation, the definition of temporal trends, and the description of cases with non-invasive tests followed by karyotype assessment. Importantly, our survey included not only amniocentesis but also a number of cases of CVS that were not described in previously published reports.

\section{Interpretation}

The observed rate of invasive testing was roughly $5 \%$, consistent with data from the large national study by Mandelbrot et al. ${ }^{9}$ from France (4.7\% in 2005-2006). Other studies had shown slightly different rates: in a study conducted in Italy between 1997 and 2003, ${ }^{6}$ the rate of invasive testing was $8.1 \%$, whereas in the sample followed by Simoes et al. ${ }^{10}$ in Portugal between 1996 and 2011, rate of amniocentesis was $3.4 \%$. Such local differences may be due to demographics of the population followed, maternal attitude toward testing, or case selection favouring a more frequent undertaking of invasive procedures in reference centres. Overall, the rate of invasive testing appears to be lower in women with HIV than in the general population in Italy: national data for 2013 showed rates of $10.6 \%$ for amniocentesis and of $4.2 \%$ for CVS, with large regional variations. ${ }^{16}$ Although the rate of invasive testing among women with HIV remained substantially unchanged between 2001 and 2015, the rate of noninvasive tests showed a significant increase during the same period that was not attributable to temporal changes in maternal age. These findings indicate a more targeted use of invasive testing based on the results of non-invasive tests, and an improved inclusion in the pathway of prenatal diagnosis in recent years.

Among the women who had biochemical screening tests followed by invasive testing, only $13 \%$ of positive screening biochemical tests were subsequently confirmed to be chromosomal anomalies at invasive testing. This finding should be further explored, considering the existing discussion about a possible higher risk of positive screening tests among women with HIV due to differences in levels of alpha-fetoprotein, human chorionic gonadotropin and unconjugated oestriol. ${ }^{17-19}$ Available information on this issue is very limited, as there is a scarcity of data on sequential non-invasive and invasive testing in pregnant women with HIV: in the study by Coll et al. ${ }^{7}$ among 10 cases with indication to amniocentesis (7/10 with a positive biochemical screening test), two (20\%) had chromosomal anomalies. Further studies, possibly based on pooling of different national cohorts of pregnant women with HIV, should specifically address this issue to avoid a potential excess of invasive testing due to increased likelihood of positive biochemical screening tests.

A confirmed prenatal diagnosis of chromosomal anomalies was frequently followed by elective termination of pregnancy. This finding is not unexpected, and consistent with the higher proportion of termination following invasive tests compared with noninvasive tests ${ }^{9}$ and following a diagnosis of chromosomal anomalies compared with structural defects. $^{20}$

A known adverse consequence of invasive procedures is fetal death. In our study, three cases of intrauterine deaths were observed $(2.6 \%)$. One of these cases was considered possibly to be related to the procedure because invasive testing was complicated by development of chorioamnionitis in the absence of other predisposing conditions, whereas the other two were considered unrelated because of congenital defects and HELLP syndrome, respectively. Variable rates have been reported for intrauterine death in women with HIV undergoing invasive procedures: Mandelbrot et al. ${ }^{9}$ observed a low rate $(0.6 \%)$, with no substantial difference in the proportion of perinatal death between cases with and without invasive testing $(0.9 \%$ versus $1.2 \%)$, whereas in the study by Somigliana et al. $^{6}$ the rate of intrauterine death was higher (5\%), with one case considered to be related to the invasive procedure. Such findings are difficult to interpret, given the limited information and the variable rates of intrauterine death reported in the general population of women with HIV. Early studies, conducted in a context of high maternal viral load/ low CD4 cell count and no use of HAART, had shown high rates $(11 \%),{ }^{21}$ but more recent studies have shown much lower rates $(1.9 \%)$. $^{22}$ Advanced maternal disease or high plasma viral load may represent significant predictors of this outcome and explain the above differences. ${ }^{23,24}$

In terms of HIV transmission, the rate observed was $2.3 \%$, with no cases observed after 2005 and no transmission events among women on HAART. Overall, these findings are consistent with data from two other European studies that also showed no transmission among the women who were on HAART at the time of the procedure ${ }^{9,10}$ and with the less recent Italian study, where the two cases of transmission that followed invasive testing (3.3\%) involved one woman not on treatment and one woman on zidovudine monotherapy. ${ }^{6}$

HIV transmission may occur independently of the invasive diagnostic test conducted, and other factors might play a 
role. A timely diagnosis of HIV in pregnancy is essential to avoid invasive testing being performed in women with unsuppressed viral replication because of undiagnosed HIV infection. Even in the presence of a negative HIV test at entry in pregnancy, particular attention should be paid to identifying HIV seroconversion in pregnancy, because of the higher risk of transmission associated with this occurrence. One of the two cases in our study involved primary infection, as well as the only observed case among the women with amniocentesis followed by Simoes et al. ${ }^{10}$ between 1996 and 2011. Finally, we observed a minimal rate of vaginal delivery among women with amniocentesis or CVS. This finding is partly due to an overall low rate in the cohort (overall, roughly 4-5\% between 2001 and 2016), compared with other European countries, ${ }^{25}$ but it could also be due to preferred elective caesarean delivery, for unclear reasons, among women who underwent invasive testing.

\section{Conclusion}

Our data reinforce the assumption that invasive prenatal testing does not increase the risk of vertical transmission in $\mathrm{HIV}$-infected pregnant women under suppressive antiretroviral treatment. Invasive procedures, however, also carry some risk of other adverse pregnancy outcomes, particularly fetal death, which should always be considered. In the future, following further evaluation and validation in the general population, prenatal diagnosis in women with HIV could also include non-invasive tests based on evaluation of fetal cell-free DNA in maternal blood, which have shown promising results in preliminary studies. ${ }^{26}$

\section{Disclosure of interests}

None declared. Completed disclosure of interests form available to view online as supporting information.

\section{Contribution to authorship}

MFl designed the study, drafted and finalised the manuscript, and was responsible for statistical analysis. GM, AM, IC, ET, AFC, SD, MS, SA, BG, AS, MCFT and MR substantially contributed to acquisition of data and critical revision of the manuscript. All the authors gave final approval to the final version to be published.

\section{Details of ethics approval}

Obtained on 28 September 2001 from the Ethics Committee of the Istituto Nazionale per la Malattie Infettive Lazzaro Spallanzani in Rome (ref. deliberation no. 578).

\section{Funding}

This work was supported by public research grants (ref.: H85E08000200005) from the Italian Medicines Agency (AIFA). No funding was received for this work from any of the following organisations: National Institutes of Health (NIH); Wellcome Trust; and the Howard Hughes Medical Institute (HHMI).

\section{Acknowledgements}

We thank Cosimo Polizzi and Alessandra Mattei of the Istituto Superiore di Sanità in Rome, Italy, for providing technical secretarial for this study. No compensation was received for this contribution.

\section{References}

1 Townsend CL, Cortina-Borja M, Peckham CS, de Ruiter A, Lyall H, Tookey PA. Low rates of mother-to-child transmission of HIV following effective pregnancy interventions in the United Kingdom and Ireland, 2000-2006. AIDS 2008;22:973-81.

2 Mandelbrot L, Tubiana R, Le Chenadec J, Doolfus C, Faye A, Pannier $E$, et al. No perinatal HIV-1 transmission from women with effective antiretroviral therapy starting before conception. Clin Infect Dis 2015:61:1715-25.

3 Chen JL, Philips KA, Kanouse DE, Collins RL, Miu A. Fertility desires and intentions of HIV-positive men and women. Fam Plann Perspect 2001;33:144-52 165.

4 Tess BH, Rodrigues LC, Newell ML, Dunn DT, Lago TD. Breastfeeding, genetic, obstetric and other risk factors associated with mother-to-child transmission of HIV-1 in Sao Paulo State, Brazil. Sao Paulo Collaborative Study for Vertical Transmission of HIV-1. AIDS 1998;12:513-20.

5 Shapiro DE, Sperling RS, Mandelbrot L, Britto P, Cunningham BE. Risk factors for perinatal human immunodeficiency virus transmission in patients receiving zidovudine prophylaxis. Pediatric AIDS Clinical Trials Group protocol 076 Study Group. Obstet Gynecol 1999;94:897-908.

6 Somigliana E, Bucceri AM, Tibaldi C, Alberico S, Ravizza M, Savasi $V$, et al. Early invasive diagnostic techniques in pregnant women who are infected with the HIV: a multicenter case series. Am J Obstet Gynecol 2005; 193:437-42.

7 Coll O, Suy A, Hernandez S, Pisa S, Lonca M, Thorne C, et al. Prenatal diagnosis in human immunodeficiency virus-infected women: a new screening program for chromosomal anomalies. Am J Obstet Gynecol 2006;194:192-8.

8 Ekoukou D, Khuong-Josses MA, Ghibaudo N, Mechali D, Rotten D. Amniocentesis in pregnant HIV-infected patients. Absence of mother-to-child viral transmission in a series of selected patients. Eur J Obstet Gynecol Reprod Biol 2008;140:212-7.

9 Mandelbrot L, Jasseron C, Ekoukou D, Batallan A, Bongain A, Pannier $E$, et al. Amniocentesis and mother-to-child human immunodeficiency virus transmission in the Agence Nationale de Recherches sur le SIDA et les Hépatites Virales French Perinatal Cohort. Am J Obstet Gynecol 2009;200:160.e1-9.

10 Simões M, Marques C, Gonçalves A, Pereira AP, Correia J, Castela J, et al. Amniocentesis in HIV pregnant women: 16 years of experience. Infect Dis Obstet Gynecol 2013;2013:914272.

11 Floridia M, Ravizza M, Tamburrini E, Anzidei G, Tibaldi C, Maccabruni A, et al. Diagnosis of HIV infection in pregnancy: data from a national cohort of pregnant women with HIV in Italy. Epidemiol Infect 2006;134:1120-7.

12 Girardi E, Vanacore P, Costa F, Solmone M, Angeletti C, Capobianchi MR, et al. Trends in HIV prevalence among pregnant women in Italy, 1994 to 2002. J Acquir Immune Defic Syndr 2006;41:361-4. 
13 Scheuerle A, Tilson $H$. Birth defect classification by organ system: a novel approach to heighten teratogenic signalling in a pregnancy registry. Pharmacoepidemiol Drug Saf 2002;11:465-75.

14 Agenzia Nazionale per i Servizi Sanitari Regionali (AGENAS): Diagnosi prenatale ed ecografia in gravidanza. Linee Guida nazionali di Riferimento - Anno 2013. [www.agenas.it/diagnosi-prenatale-edecografia-in-gravidanza]. Accessed 29 October 2015.

15 HIV/AIDS Italian Expert Panel. Linee Guida Italiane sull'utilizzo dei farmaci antiretrovirali e sulla gestione diagnostico-clinica delle persone con infezione da HIV-1. [WwW.salute.gov.it/imgs/ C_17_pubblicazioni_2261_allegato.pdf]. Accessed 26 April 2016.

16 Ministero della Salute_-Direzione Generale della digitalizzazione, del sistema informativo sanitario e della statistica. Certificato di assistenza al parto (CeDAP). Analisi dell'evento nascita_Anno 2013. [www.salute.gov.it/portale/documentazione/p6_2_2_1.jsp? lingua=italiano\&id=2431] Accessed 27 April 2016.

17 Gross S, Castillo W, Crane M, Espinosa B, Carter S, DeVeaux R, et al. Maternal serum alpha-fetoprotein and human chorionic gonadotropin levels in women with human immunodeficiency virus. Am J Obstet Gynecol 2003;188:1052-6.

18 Yudin MH, Prosen TL, Landers DV. Multiple-marker screening in human immunodeficiency virus-positive pregnant women: screen positivity rates with the triple and quad screens. Am J Obstet Gynecol 2003;189:973-6.

19 Spencer K. First and second trimester markers of fetal aneuploidy in pregnant women with HIV infection. Fetal Diagn Ther 2011;29:1358.

20 Floridia M, Mastroiacovo P, Ravizza M, Todros T, Chiadò Fiorio Tin M, Marconi AM, et al. Good prenatal detection rate of major birth defects in HIV-infected pregnant women in Italy. Prenat Diagn 2015;35:1374-8

21 Langston C, Lewis DE, Hammill HA, Popek EJ, Kozinetz CA, Kline $M W$, et al. Excess intrauterine fetal demise associated with maternal human immunodeficiency virus infection. J Infect Dis 1995; 172:1451-60.

22 Kreitchmann R, Li SX, Melo VH, Fernandes Coelho D, Watts DH, Joao $E$, et al. Predictors of adverse pregnancy outcomes in women infected with HIV in Latin America and the Caribbean: a cohort study. BJOG 2014;121:1501-8.

23 Kim HY, Kasonde P, Mwiya M, Thea DM, Kankasa C, Sinkala $M$, et al. Pregnancy loss and role of infant HIV status on perinatal mortality among HIV-infected women. BMC Pediatr 2012;12:138.

24 Cates JE, Westreich D, Edmonds A, Wriht RL, Minkoff H, Colie C, et al. The Effects of Viral Load Burden on Pregnancy Loss among HIV-Infected Women in the United States. Infect Dis Obstet Gynecol 2015;2015:362357.

25 Aebi-Popp K, Mulcahy F, Glass TR, Rudin C, Martinez de Tejada B, Bertisch B, et al. Missed opportunities among HIV-positive women to control viral replication during pregnancy and to have a vaginal delivery. J Acquir Immune Defic Syndr 2013;64:58-65.
26 Taylor-Phillips S, Freeman K, Geppert J, Agbebiyi A, Uthman OA, Madan J, et al. Accuracy of non-invasive prenatal testing using cellfree DNA for detection of Down, Edwards and Patau syndromes: a systematic review and meta-analysis. BMJ Open 2016;6:e010002.

\section{Appendix 1}

\section{The Italian Group on Surveillance of Antiretroviral Treatment in Pregnancy}

Project coordinators: M. Floridia, M. Ravizza, E. Tamburrini.

Participants: M. Ravizza, E. Tamburrini, F. Mori, P. Ortolani, E.R. dalle Nogare, F. Di Lorenzo, G. Sterrantino, M. Meli, S. Polemi, J. Nocentini, M. Baldini, G. Montorzi, M. Mazzetti, P. Rogasi, B. Borchi, F. Vichi, B. Del Pin, E. Pinter, E. Anzalone, R. Marocco, C. Mastroianni, V.S. Mercurio, A. Carocci, E. Grilli, A. Maccabruni, M. Zaramella, B. Mariani, G. Natalini Raponi, G. Guaraldi, G. Nardini, C. Stentarelli, B. Beghetto, A.M. Degli Antoni, A. Molinari, M.P. Crisalli, A. Donisi, M. Piepoli, V. Cerri, G. Zuccotti, V. Giacomet, S. Coletto, F. Di Nello, C. Madia, G. Placido, A. Vivarelli, P. Castelli, F. Savalli, V. Portelli, F. Sabbatini, D. Francisci, L. Bernini, P. Grossi, L. Rizzi, S. Alberico, G. Maso, M. Airoud, G. Soppelsa, A. Meloni, M. Dedoni, C. Cuboni, F. Ortu, P. Piano, A. Citernesi, I. Bordoni Vicini, K. Luzi, A. Spinillo, M. Roccio, A. Vimercati, A. Miccolis, A. De Gennaro, B. Guerra, F. Cervi, G. Simonazzi, E. Margarito, M.G. Capretti, C. Marsico, G. Faldella, M. Sansone, P. Martinelli, A. Agangi, A. Capone, G.M. Maruotti, C. Tibaldi, L. Trentini, T. Todros, G. Masuelli, V. Frisina, I. Cetin, T. Brambilla, V. Savasi, C. Personeni, C. Giaquinto, M. Fiscon, E. Rubino, A. Bucceri, R. Matrone, G. Scaravelli, O. Genovese, C. Cafforio, C. Pinnetti, G. Liuzzi, V. Tozzi, P. Massetti, A.M. Casadei, A.F. Cavaliere, M. Cellini, G. Castelli Gattinara, A.M. Marconi, S. Dalzero, V. Sacchi, M. Ierardi, C. Polizzi, A. Mattei, M.F. Pirillo, R. Amici, C.M. Galluzzo, S. Donnini, S. Baroncelli, M. Floridia.

Pharmacokinetics: P. Villani, M. Cusato.

Advisory Board: A. Cerioli, M. De Martino, P. Mastroiacovo, F. Parazzini, E. Tamburrini, S. Vella.

SIGO-HIV Group National Coordinators: P. Martinelli, M. Ravizza. 